Objectives Low wage and immigrant workers suffer an excess of injuries and are often difficult to reach. There is a need to identify alternative forums for health and safety training. The aim of this study was to determine whether injured workers cluster by geographic area; this would serve as a basis for targeting occupational health and safety interventions at the community level.

Method Work-related injuries from the Illinois Trauma Registry were extracted and mapped by residential zipcode for 20002009. Injury data was merged with employment data.

Results There were 23200 work-related injuries. Of the 1382 zipcodes, $79.3 \%$ of the injuries occurred among residents living in $20 \%$ of the zipcodes. $21.2 \%$ of the work-related injuries $(\mathrm{N}=4914)$ occurred in the 25 zipcodes with the highest counts. We identified six spatial clusters. In the 25 ZIP codes with the highest rates of injuries among employed persons, less than $1 \%$ of the injuries occurred in these zipcodes $(\mathrm{N}=99)$.

Conclusions Training at the community level could reduce workplace injury and is needed to augment the void in workplace training. Alternative datasets, not originally designed for occupational surveillance, could be useful for identifying communities with occupational injury and illness clusters. The validity and usefulness of these datasets should be further assessed, and the communities in which these clusters occur should be mapped to identify community level infrastructure that could be leveraged for training interventions. A bridge should be created between occupational medicine, governmental institutions, social work and community advocacy in order to make a community intervention program viable.

\section{A CASE-CONTROL STUDY OF OCCUPATIONAL EXPOSURE TO METALWORKING FLUIDS AND BLADDER CANCER RISK AMONG MEN}

${ }^{1}$ Joanne Colt, ${ }^{1}$ Melissa Friesen, ${ }^{2}$ Patricia Stewart, ${ }^{3}$ Park Donguk, ${ }^{4}$ Alison Johnson, ${ }^{5}$ Molly Schwenn, ${ }^{6}$ Margaret Karagas, ${ }^{7}$ Karla Armenti, ${ }^{6}$ Richard Waddell, ${ }^{5}$ Castine Verrill, ${ }^{1}$ Mary Ward, 'Laura Beane Freeman, 'Lee Moore, 'Dalsu Baris, ${ }^{1}$ Debra Silverman. ${ }^{1}$ Division of Cancer Epidemiology and Genetics, National Cancer Institute, National Institutes of Health, Department of Health and Human Services, Bethesda, MD, USA; ${ }^{2}$ Stewart Exposure Assessments, LLC, Arlington, VA, USA; ${ }^{3}$ Korea National Open University, Seoul, Republic of Korea; ${ }^{4}$ Vermont Cancer Registry, Burlington, VT, USA; ${ }^{5}$ Maine Cancer Registry, Augusta, ME, USA; ${ }^{6}$ Geisel School of Medicine at Dartmouth, Hanover, NH, USA; ${ }^{7}$ New Hampshire Department of Health and Human Services, Concord, NH, USA

\subsection{6/oemed-2014-102362.221}

Objectives Metalworking has been associated with bladder cancer risk in many studies. Metalworking fluids (MWFs) are suspected as the putative exposure, but epidemiologic data are limited. Based on state-of-the-art, quantitative exposure assessment, we examined MWF exposure and bladder cancer risk in the New England Bladder Cancer Study.

Method Male cases $(\mathrm{n}=895)$ and population controls $(\mathrm{n}=$ 1031) provided occupational histories and information on use of each of three MWF types: (1) straight (mineral oil, additives), (2) soluble (mineral oil, water, additives), and (3) synthetic (water, organics, additives) or semi-synthetic (soluble/ synthetic hybrid), in response to exposure-oriented modules administered during personal interviews. We estimated the probability, frequency, and intensity of exposure to each MWF type and, if probability exceeded 50\%, cumulative exposure. Logistic regression was used to calculate odds ratios (ORs) and
95\% confidence intervals (CIs), adjusting for smoking and other risk factors.

Results Risk was increased for men reporting use of straight MWFs $(\mathrm{OR}=1.7,95 \% \mathrm{CI}=1.1-2.8)$, with a significant trend with increasing cumulative exposure $(\mathrm{p}=0.024)$. Use of soluble MWFs conferred a $50 \%$ elevated risk $(95 \% \mathrm{CI}=0.96-2.5)$. ORs were nonsignificantly elevated for synthetic MWFs, based on small numbers. Men who were never metalworkers, but held jobs with possible exposure to mineral oil, had a $40 \%$ increased risk $(95 \% \mathrm{CI}=1.1-1.8)$.

Conclusions In the most comprehensive assessment of MWF exposure in a bladder cancer case-control study, exposure to straight MWFs significantly increased bladder cancer risk, as did employment in non-metalworking jobs with possible mineral oil exposure. Our results strengthen prior evidence for mineral oil as a bladder carcinogen.

\section{PSYCHOSOCIAL WORKING ENVIRONMENT FOR PATIENTS WITH ISCHAEMIC HEART DISEASE AND ASSOCIATION TO ADVERSE CARDIAC EVENTS}

${ }^{1}$ Karin Biering, ${ }^{2,3}$ Thomas Lund, 'Johan Hviid Andersen, ${ }^{4,5}$ Niels Henrik Hjollund. 'Danish Ramazzini Centre, University Department of Occupational Medicine, Herning, Denmark; ${ }^{2}$ National Centre for Occupational Rehabilitation, Rauland, Norway; ${ }^{3}$ MarselisborgCentret, Research and Development, Public Health and Quality Improvement, Central Denmark Region, Aarhus, Denmark; ${ }^{4}$ WestChronic, Herning, Denmark; ${ }^{5}$ Department of Clinical Epidemiology, Aarhus, Denmark

\subsection{6/oemed-2014-102362.222}

Objectives During the last decades a possible association between the psychosocial working environment and increased risk of Ischaemic heart disease (IHD) has been debated. A systematic review from 2009 found moderate evidence that high psychological demands, lack of social support and iso-strain was associated with IHD. Whether the psychosocial working environment plays a role for patients with existing cardiovascular disease on the risk of new cardiac events and readmissions is unknown

Method A cohort of patients under 65 years and treated with Percutaneous Coronary Intervention was established in 2006. Three months after the procedure the patients answered a questionnaire about their psychosocial working environment. A total of 528 patients had returned to work 12 weeks after the procedure, while 97 were still sick-listed. Patients were followed in registers for $3+$ years to determine cardiac readmissions and events. We examined the association between psychosocial working environment and adverse events among those who had returned to work at 3 months by Cox Regression analysis.

Results We were not able to detect any significant associations between psychosocial working environment in terms of quantitative and cognitive demands, workload, involvement, influence, tolerance, social support, the combinations of effort-reward and demand-control and the risk of adverse events.

Conclusions Reporting of problems in the psychosocial working environment are not associated with risk of adverse cardiac events. However, tendencies of a lower risk of cardiac event were present for employees reporting the worst psychosocial environment. This unexpected finding may be explained by vulnerable persons not returning to work. 